\title{
Multimodality Review of Imaging Features Following Breast Reduction Surgery
}

\author{
Sally H. Goudreau ${ }^{1}$, (1) Meghan A. Woughter ${ }^{2}$, (1) Stephen J. Seiler ${ }^{1}$ \\ ${ }^{1}$ Department of Radiology, UT Southwestern Medical Center, Texas, USA \\ ${ }^{2}$ Department of Radiology, Radiology Partners, Texas, USA
}

\begin{abstract}
Reduction mammoplasty is a common surgical procedure that removes a significant portion of the breast, and the resulting changes to the breast parenchyma are frequently seen on breast imaging studies. Any radiologist who interprets breast imaging studies must be able to recognize these changes in order to avoid unnecessary recall from screening and/or breast biopsy. The surgical techniques used in reduction mammoplasty are discussed in order to provide relevant background information for understanding the resulting imaging features. These imaging characteristics are presented for the most common breast imaging modalities, including mammography, ultrasound, and magnetic resonance imaging. Additionally, tips for distinguishing malignancy from postsurgical change are provided, as are potential pitfalls in imaging interpretation. To avoid unnecessary patient morbidity, it is critical to differentiate between the classic, benign imaging appearance of the breast after reduction mammoplasty and findings that indicate a potential malignancy.
\end{abstract}

Keywords: Breast cancer, breast imaging, breast reconstruction, mastopexy

Cite this article as: Goudreau SH, Woughter MA, Seiler SJ. Multimodality Review of Imaging Features Following Breast Reduction Surgery. Eur J Breast Health 2021; 17(3): 206-213

\section{Key Points}

- All reduction procedures involve the removal and displacement of varying amounts of parenchymal tissue and skin, largely from the inferior breast.

- This tissue and skin removal alters the normal distribution of fibroglandular tissue and can result in architectural distortion, focal asymmetries, and regions of fat necrosis with or without dystrophic calcifications.

- Fat necrosis may be difficult to interpret by ultrasound interrogation alone. A spot tangential mammographic view may assist in the visualization of internal fat within the mass to clench the diagnosis of fat necrosis.

- Preoperative imaging with mammography is recommended to assess for occult malignancy for average-risk women 40 years of age and older as well as women of any age who are at high-risk of developing breast cancer.

\section{Introduction}

Reduction mammoplasty is a common non-oncologic surgical procedure that accounts for $18 \%$ of all breast surgeries (1). In patients with symptomatic macromastia, the surgery aims to remove excess fat, glandular tissue, and skin from the breast to create a smaller, more aesthetically pleasing breast shape (2). Moreover, chronic back, neck, or shoulder pain, kyphosis, chronic intertrigo involving the inframammary folds, limitation of physical activity, or other problems associated with overly large breasts are common indications. Following reduction surgery, preoperative symptoms have been shown to be significantly reduced (3). In addition, some patients have reduction mammoplasty to improve symmetry following a contralateral partial mastectomy or total mastectomy with reconstruction. Several similar surgical techniques are used to alleviate patient symptoms while producing aesthetically pleasing results. Disruption of breast tissue following these surgical procedures can appear perplexing to those who are unfamiliar with these classic changes. Once the technical aspects of these procedures are conceptualized, the imaging appearance of the breast frequently becomes apparent. Even an experienced interpreting radiologist may have difficulty distinguishing between postsurgical changes after reduction mammoplasty and the imaging appearance of malignancy on occasion. Ultimately, if malignancy is suspected, a biopsy will be required for a definitive histopathologic diagnosis. 


\section{Surgical techniques}

There are several reduction mammoplasty techniques available, the majority of which involve removing parenchymal tissue and skin from the inferior breast while elevating the nipple-areolar complex (NAC). The most common surgical techniques for reduction mammoplasty involve a circumferential incision around the NAC, followed by a vertical incision extending to the inframammary fold, resulting in a keyhole scar (Figure 1a). This technique enables superior repositioning of the NAC as well as removal and repositioning/lifting of the inferior breast tissue (mastopexy). By using this keyhole skin flap pattern, the conical breast shape is preserved because the skin flaps are opposed, resulting in a skin brassiere. To achieve an aesthetic result, the NAC can be transposed with the underlying ducts and vascular pedicle intact using the inferior pedicle technique (Figure 1b) or transplanted with a full-thickness free nipple-areolar graft. With the inferior pedicle technique, the blood supply to the nipple and areola originates from the relatively large surface of the inframammary fold, which contributes to the preservation of nipple, areola, and skin sensations (4). Because it has a lower risk of NAC avascular necrosis than NAC transposition, free nipple-areolar graft transplantation is more common in women with large, pendulous breasts and those past childbearing age (5). The resulting post-reduction skin scar usually has a keyhole or lollipop appearance, with a subtle periareolar scar, a vertical scar extending inferiorly from the nipple to the inframammary fold, and a transverse scar curving along the fold (Figure 1c).

Breast reduction can also be accomplished solely through liposuction. This is the least invasive technique, resulting in only minor volume reduction and omitting the benefit of mastopexy. There are some non-specific imaging findings associated with this technique, such as developing asymmetries in regions where fat has been removed. Obtaining a history of the plastic surgical intervention would be beneficial in avoiding a biopsy of the new imaging finding, which would otherwise be required.

\section{Mammography}

\section{Architectural distortion}

When evaluating architectural distortion in the breast, the presence of surgical clips is often the first indication that a patient has had a prior surgical procedure, though this does not always mean reduction mammoplasty. While some surgical clips are left behind in reduced breasts, most surgeons do not. When mammographically evaluating what is thought to be postsurgical architectural distortion, the clinical history is confirmatory.

All reduction procedures involve the removal and displacement of varying amount amounts of parenchymal tissue and skin, which alters the normal distribution of fibroglandular tissue and can result in focal asymmetries (Figure 2a). In one early study of post-reduction mammographic changes, these asymmetries developed in roughly half of the women postoperatively, either persisting or gradually decreasing over time (6). With the appropriate clinical history and knowledge of this typical mammographic appearance, unnecessary recall from screening and/or biopsy can be avoided.

During reduction mammoplasty, most breast tissue is usually excised from the inferior aspect of the breast. The remaining tissue is gathered together, often with rearrangement. This technique produces a distinctive pattern of architectural distortion that appears swirled or with an upward sweeping configuration (Figure 2b) (5). Mammographically, such inferior pole changes are evident in nearly all patients $(6,7)$.

Typical post-reduction scar patterns also include fibrotic bands that run parallel to the skin scar line and are easier to identify in non-anatomic orientations. Vertical scarring is common in the inferior breast, and
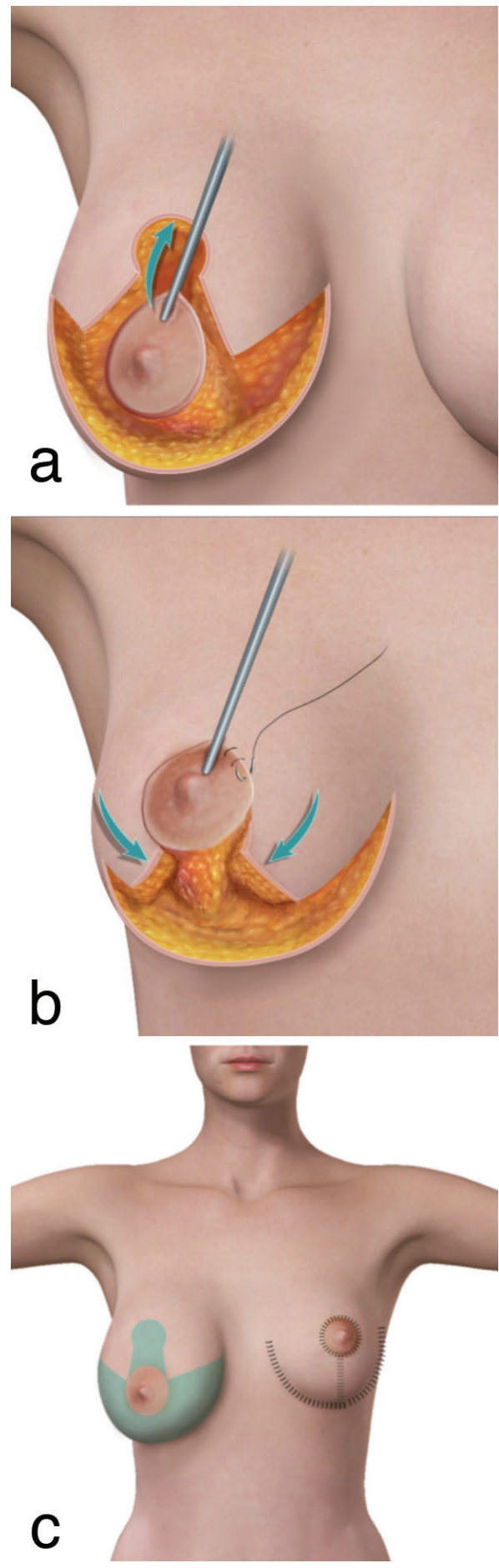

Figure 1. Surgical incisions for reduction mammoplasty. (a) An incision is made around the nipple areolar complex along with a vertical incision extending to a curvilinear inframammary fold incision. (b) The breast tissue is removed inferiorly, and the nipple is transposed superiorly, maintaining the vascular pedicle. (c) Appearance of the breast before (right breast) and after (left breast) reduction mammoplasty using the keyhole technique 
transversely oriented scars can be seen posteriorly in the breast (8). This non-anatomic scarring pattern may result in linear bands that mammographically resemble skin folds (Figure 2c). These bands can be subtle, thinning over time and becoming difficult to distinguish
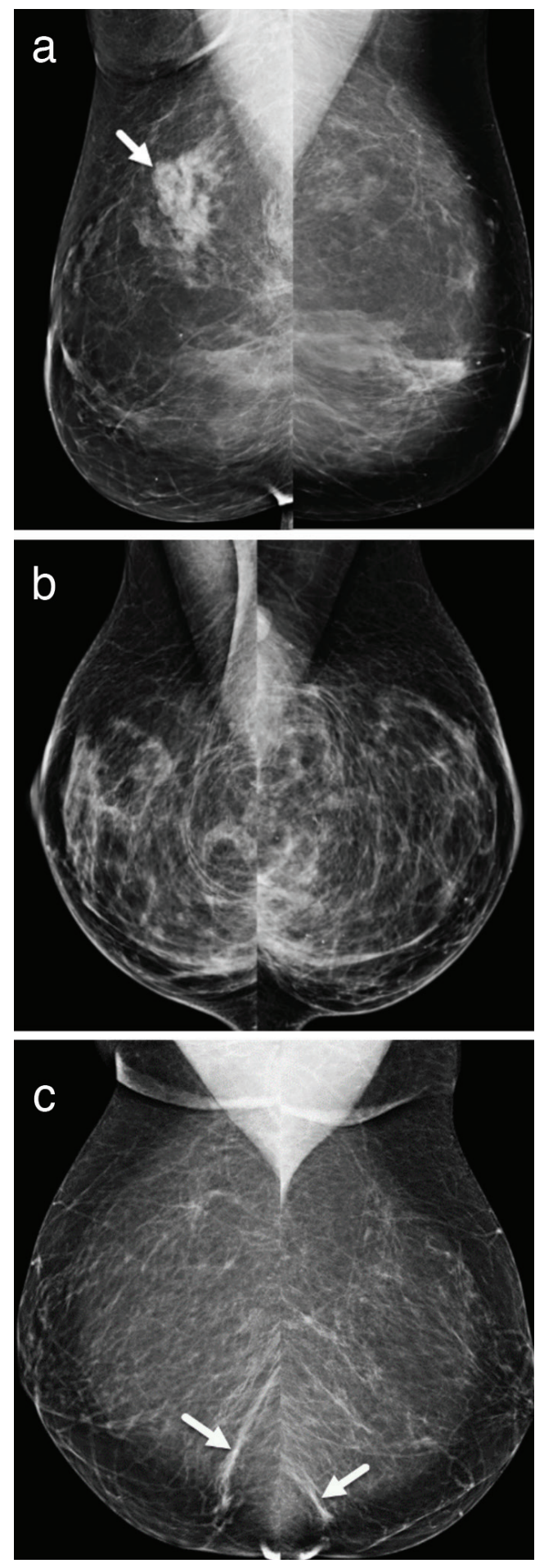

Figure 2. Rearrangement of the fibroglandular breast tissue after reduction mammoplasty. (a) Bilateral mediolateral oblique digital mammographic images show asymmetric tissue in the right posterior superior breast resulting from tissue rearrangement following mammoplasty. (b) Bilateral mediolateral oblique digital mammograms show the characteristic sweeping parenchymal pattern seen after breast reduction. (c) Bilateral mediolateral oblique digital mammograms demonstrate non-anatomic post-reduction from normal breast parenchyma. Parenchymal bands, which can extend from the chest wall to the NAC, can also be relatively thick. The most common cause of non-malignant architectural distortion is postsurgical scarring. However, as with any type of breast surgery, parenchymal surgical scarring should diminish or stabilize over time. New or increasing architectural distortion, even scarred regions, is suspicious and warrants further investigation (Figure 3).
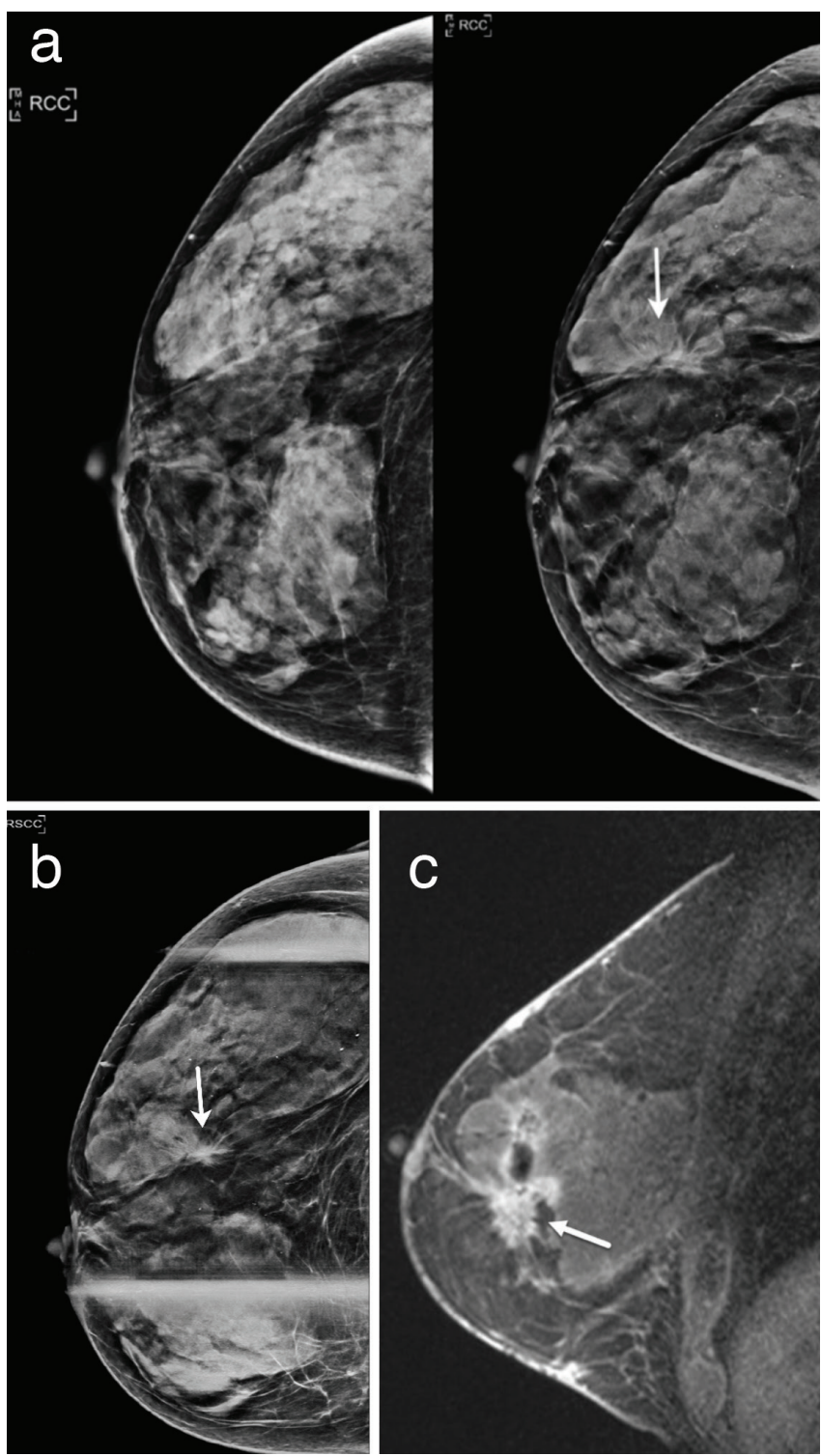

Figure 3. Malignancy developing in a reduction scar. (a) Sequential right craniocaudal images taken one year apart as part of screening examinations in a 57-year-old woman demonstrate interval development of architectural distortion at the reduction scar (arrow). (b) Craniocaudal right spot compression image taken as part of a diagnostic work-up shows persistent architectural distortion (arrow). Sonography at that time was non-contributory, only revealing scarring in the area of concern. Stereotactic core biopsy of the architectural distortion (not shown) confirmed invasive ductal carcinoma. (c) Enhanced MRI was subsequently performed to evaluate the extent of disease which demonstrated extensive multifocal/multicentric abnormal enhancement, including the area of developing architectural distortion (arrow) seen on this sagittal image of the right breast

MRI: Magnetic resonance imaging 


\section{Changes of the NAC}

Mastopexy is used in various types of reduction mammoplasty procedures, with the NAC being relocated superiorly, as seen on the mediolateral oblique views. To create a better periareolar scar, permanent suture material may be used to secure the nipple complex (Figure 4). Along the periareolar margin, scarring with or without calcifications may be visible (9). Danikas et al. (7) demonstrated that these periareolar alterations can be seen on mammography in $85 \%$ of women postoperatively.

\section{Calcifications and fat necrosis}

Benign calcifications are a common postoperative mammographic finding, though they appear later than other mammographic features. According to one study, calcifications were found in only $3 \%$ of mammograms performed within the first 12 months after reduction, compared to $53 \%$ of mammograms performed 24 months or later after surgery (6). Furthermore, skin calcifications with lucent centers are more common at anastomotic sites.

Breast reduction surgery usually entails extensive manipulation of the breast parenchyma. Fat necrosis is caused by major trauma to an area of adipose tissue, which results in cellular death of the adipocytes and the subsequent appearance of residual oil/fat material and dystrophic calcification. As a result, fat necrosis is often encountered postoperatively and is a common cause of palpable abnormality in the postoperative breast (10). Because of internal fat at the palpable site that correlates well with the clinical history, a new palpable area of fat necrosis is often easily diagnosed mammographically, whereas the corresponding sonographic appearance can be indeterminate and potentially suspicious (Figure 5).

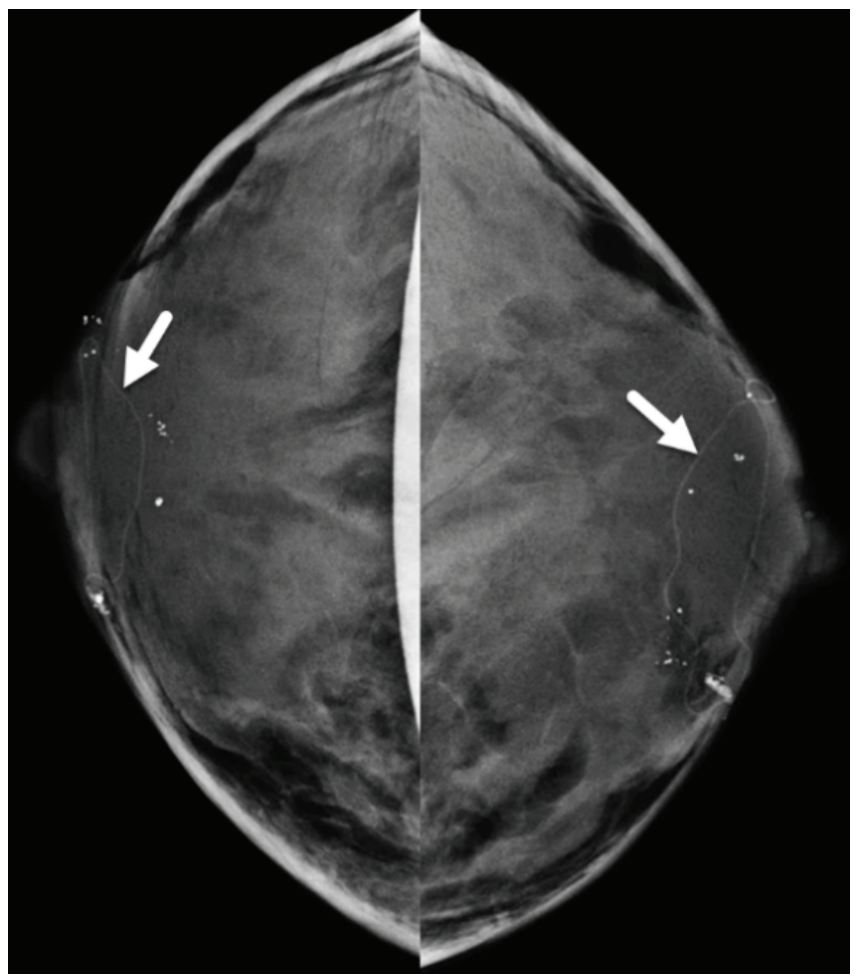

Figure 4. Mammographic appearance of the nipple areolar complex after reduction mammoplasty. Permanent sutures are seen (arrows) in the peri-areolar regions on bilateral craniocaudal digital mammographic images
Breast surgery frequently results in areas of benign fat necrosis manifesting as oil cysts. These masses are well-defined, are round or oval in shape, and contain fat, with or without rim calcification ( 5 , 11 ). Oil cysts of varying sizes are seen in nearly $20 \%$ of patients and may resolve or shrink in size over time (7). As a result of oil cysts, dystrophic calcifications can develop. These calcifications may be difficult to interpret accurately at first, but they frequently coarsen over time.

Fat necrosis can also manifest as a nonspecific mass or a focal architectural distortion with or without calcifications. Moreover, postoperative changes and fat necrosis can easily be attributed to fat-containing masses with or without associated coarse or rim calcifications. However, dystrophic calcifications associated with fat necrosis that appear in the early postoperative stage infrequently may have a questionable morphology and/or distribution (12). The risk of malignancy is still very low in these cases, and calcifications associated with fat necrosis should gradually evolve, assuming a more classic dystrophic appearance and confirming the benign etiology (12). Therefore, reporting these calcifications in the Breast Imaging Reporting and Database System 3 (BI-RADS 3) category (0\% to $\leq 2 \%$ likelihood of malignancy) with a recommendation for a short-
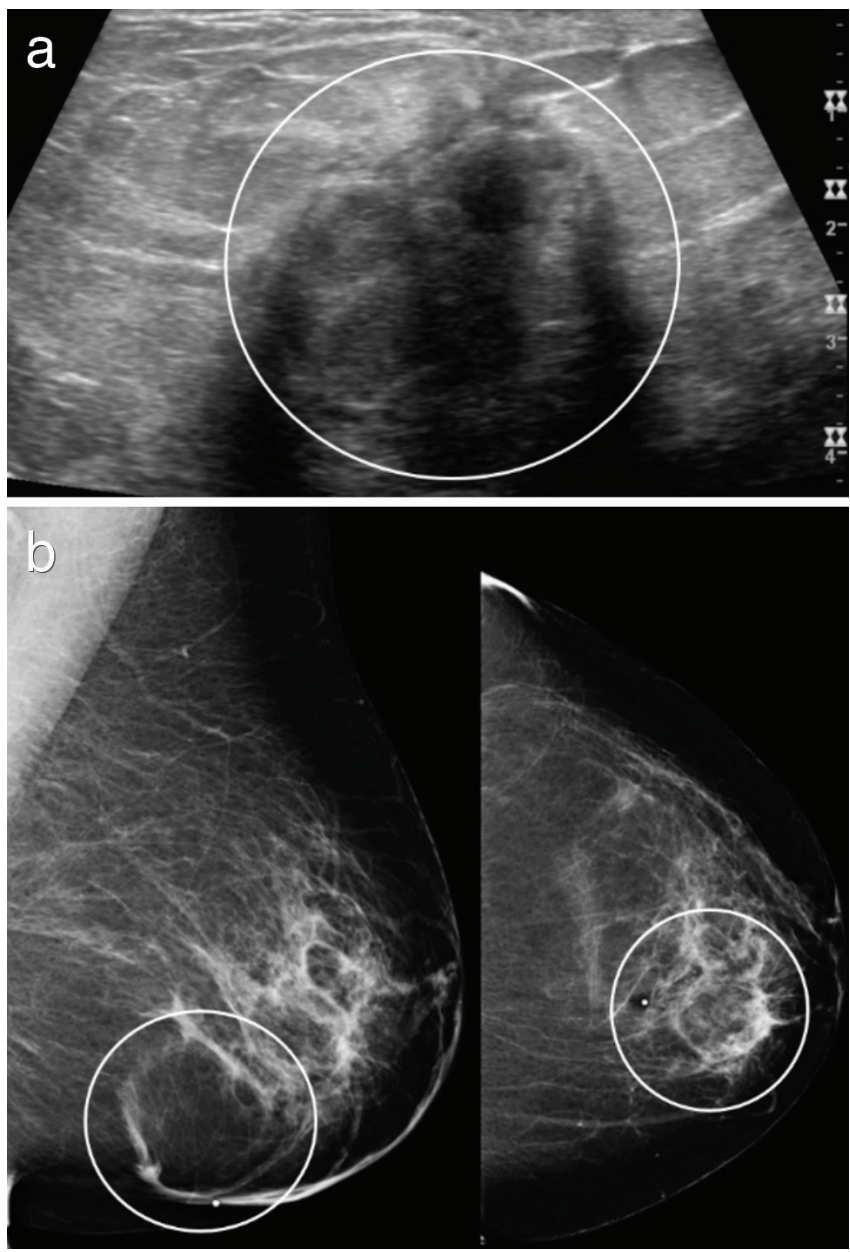

Figure 5. Post-reduction fat necrosis presenting as an area of palpable concern. (a) Sonography shows a nonspecific, solid, irregularly shaped heterogeneous mass (circle) in the region of a new, palpable lump. (b) Left mediolateral oblique and craniocaudal digital mammogram images subsequently performed demonstrate post-reduction changes with a fatty mass (circles) in the 7:00 position, confirming benign fat necrosis 
term close interval follow-up to document morphologic stability is appropriate. Stereotactic biopsy should be reserved for cases that do not show early morphology and/or distribution of fat necrosis, or for cases that become suspicious after a short interval of follow-up.

\section{Sonography}

Physical examination of the breast, along with sonography, will reveal the typical scarring pattern associated with prior reduction mammoplasty. A periareolar skin scar (which may be very subtle), an inframammary fold scar, and a radially oriented scar in the 6 o'clock position will all be visible patterns. Depending on the type of surgical procedure performed, patients may have a variable combination of these scars.

The appearance of post-reduction fat necrosis on imaging varies greatly depending on its stage of evolution, particularly with sonography. Sonography is frequently used to investigate new palpable findings; however, fat necrosis is can be difficult to interpret with ultrasound alone. If the etiology of a palpable mass cannot be determined after an initial ultrasound interrogation, a spot tangential mammographic view can be obtained easily in order to visualize fat within the mass (Figure 6). One of the most useful features in classifying the mass as benign fat necrosis is the discovery of internal fat.
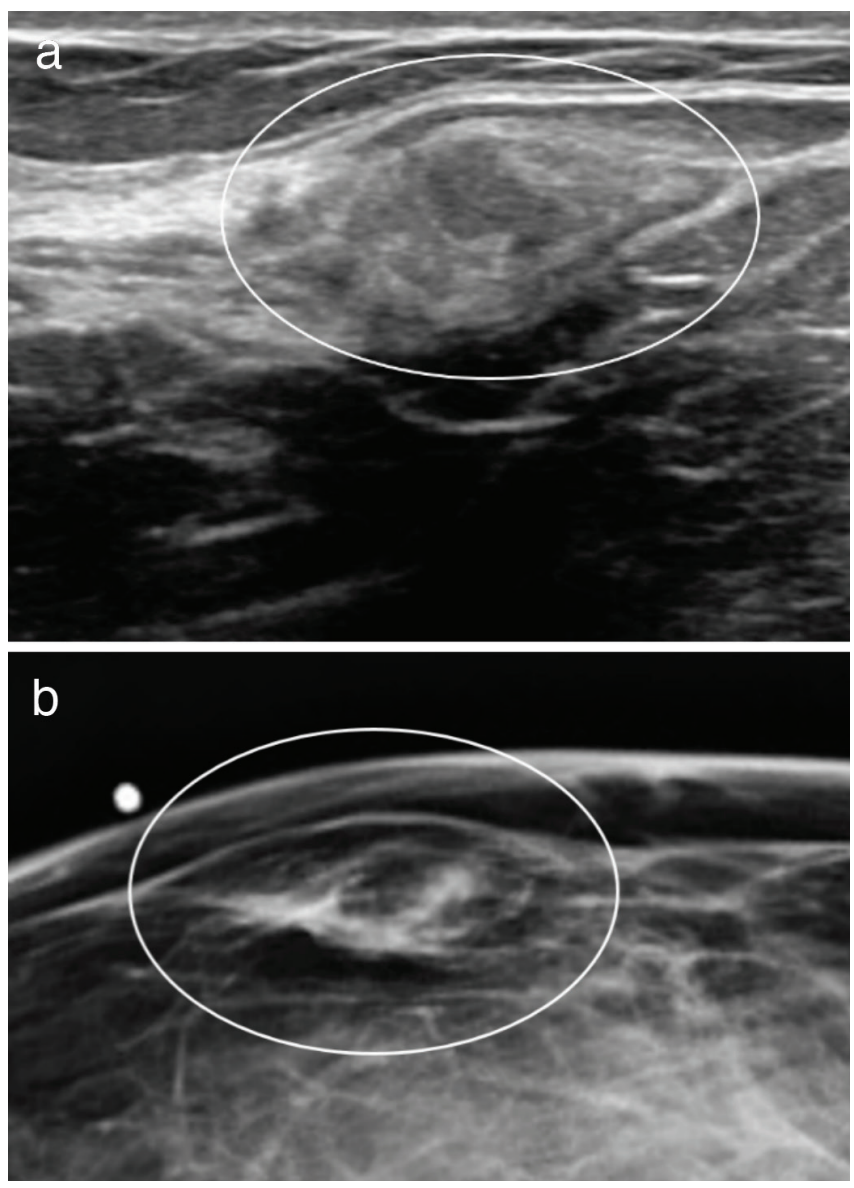

Figure 6. A palpable mass seen sonographically is confirmed to be fat necrosis with mammography. (a) Sonographic image shows an irregularly shaped, heterogeneous mass (circle) at the site of a patient's palpable abnormality after reduction mammoplasty. (b) Corresponding spot tangential digital mammogram of the palpable finding demonstrates a fat containing mass (circle), confirming fat
Furthermore, architectural distortion can occur anywhere within the breast parenchyma in post-reduction patients if significant tissue rearrangement has occurred. Sonographically, this distortion appears as vague hypoechogenicity, disruption of the normal fascial planes, and posterior acoustic shadowing, which is most prominent in the inferior breast and inframammary fold region. This sonographic appearance may be identical to cancer, necessitating a biopsy.

\section{Magnetic resonance imaging}

Because of its high sensitivity and negative predictive value of malignancy, breast magnetic resonance imaging is a valuable diagnostic tool for detecting breast cancer. There are a number of distinct postreduction mammoplasty findings seen with magnetic resonance imaging (13).

Signal voids corresponding to suture material and/or surgical clips are seen in a linear fashion along the inframammary fold, encircle the NAC, and may be scattered throughout the breast parenchyma (Figure 7a). Similarly, post-contrast imaging can easily detect dermal scarring and keloids around the NAC, inframammary fold, and 6 o'clock radiant (Figure $7 \mathrm{~b}$ ). During surgery, fibroglandular tissue is rearranged, resulting in architectural distortion, parenchymal bands (Figure 7c), and islands of breast tissue (Figure 7d) similar to those seen on mammography. The imaging characteristics of these islands should be similar to other areas of benign fibroglandular tissue found in the breast.

Fat necrosis can occur anywhere in the reconstructed breast, but it is most common along the inferior aspect of the breast, where distortion is most common. Fat necrosis produces an isointense signal to the rest of the fatty breast tissue, with varying degrees of rim enhancement depending on its current stage of evolution and the degree of inflammation and granulation tissue. Although a thin enhancing rim is common with fat necrosis, a thickened and irregular enhancing rim that can be mistaken for malignancy may be present. The kinetic analysis of fat necrosis is nonspecific, encompassing both benign and malignant enhancement patterns (13). T1-weighted images with and without fat saturation are frequently used to determine the presence of fat within a mass or area of architectural distortion, assisting in the confirmation of the presence of benign fat necrosis (Figure 8). Fat necrosis may also be characterized by enhancing internal thin septations. The T1 signal from fat necrosis is typically isointense to other fats in the breast; however, fat necrosis may sometimes appear to have a slightly darker T1 signal due to hemosiderin deposition or chronic inflammatory changes. Mammographic correlation is recommended because the presence of oil cysts or coarse calcifications within the region of interest may provide further supporting evidence of fat necrosis.

\section{Cancer detection}

Prior to undergoing elective reduction mammoplasty, preoperative imaging to assess for occult malignancy is recommended for averagerisk women 40 years of age and older, as well as women of any age who are at high-risk of developing breast cancer (14). Mammography is the most cost-effective and widely available screening method in eligible patients. Any suspicious lesions detected during preoperative imaging will require tissue diagnosis prior to surgery. Infrequently $(0.8 \%)$, malignancy is discovered during reduction mammoplasty, posing a diagnostic dilemma if no preoperative imaging was 
obtained (15). In the event of a postoperative diagnosis of breast cancer, the patient will need additional diagnostic evaluation; however, the sensitivity of breast magnetic resonance imaging for malignancy is reduced in the immediate post-reduction breast due to the expected postoperative enhancement of inflamed and healing tissue. In a large cohort of 4,804 women examined by Tang et al. (15), $48 \%$ of patients with an incidental diagnosis of malignancy at mammoplasty had postoperative breast magnetic resonance imaging to assess the extent of disease. The majority of the initial cancers discovered were low grade and small. In fact, $8 \%$ of invasive cancers and $72 \%$ of DCIS were grade 1 or 2 , and $94 \%$ of invasive cancers were stage $\mathrm{T} 1$. The authors found that postoperative magnetic resonance imaging had limited sensitivity for detecting any residual malignancy, hypothesizing that this was due to the small size of any
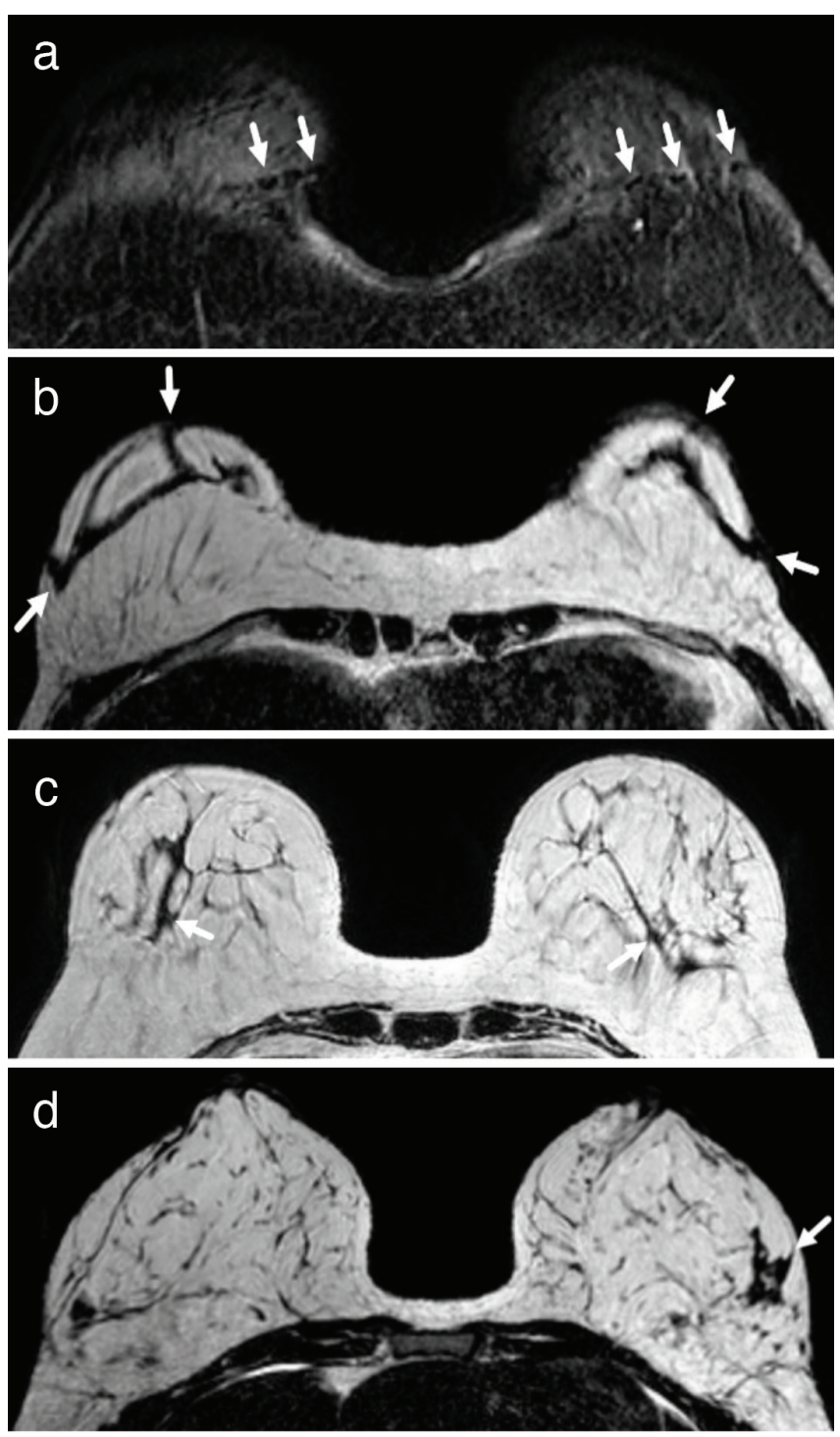

Figure 7. Post-surgical magnetic resonance appearance after reduction mammoplasty. (a) Signal void along the bilateral inframammary folds on a fat saturated T1-weighted axial image corresponds to surgical staples and/or sutures (arrows). (b) Scarring is represented by dark bands (arrows) on a T1-weighted axial image along the inframammary fold region bilaterally. (c) A T1-weighted axial image shows parenchymal bands (arrows) similar to those seen mammographically. (d) An axial post-contrast image shows an island of non-enhancing fibroglandular tissue (arrow) in the lateral left breast residual cancer and masking from postsurgical changes (15). At our institution, we have observed similar limitations in the immediate postoperative period and therefore recommend that the patient wait at least 6 weeks after surgery before undergoing magnetic resonance imaging to evaluate for residual disease in order to lessen these postoperative changes. After treatment for the primary malignancy, if mastectomy is not performed, repeat bilateral breast magnetic resonance imaging in 6 months could be considered for reevaluation after the postsurgical changes have resolved. Cancer detection and recall rates in breasts that have undergone reduction mammoplasty have been reported to be comparable to native breast (16). For average-risk women, routine annual or biennial mammographic screening following reduction mammoplasty is the appropriate recommendation. The postoperative baseline mammogram is usually the most difficult to interpret because significant changes in the configuration of the breast parenchyma have occurred in addition to the expected interval postoperative changes (7). Any interval changes, such as a developing asymmetry or mass, after this baseline mammogram should be viewed with caution, and an appropriate diagnostic evaluation should be recommended (Figure 9).

Differentiating fat necrosis from residual or new cancer can sometimes be difficult with any breast imaging modality, especially if
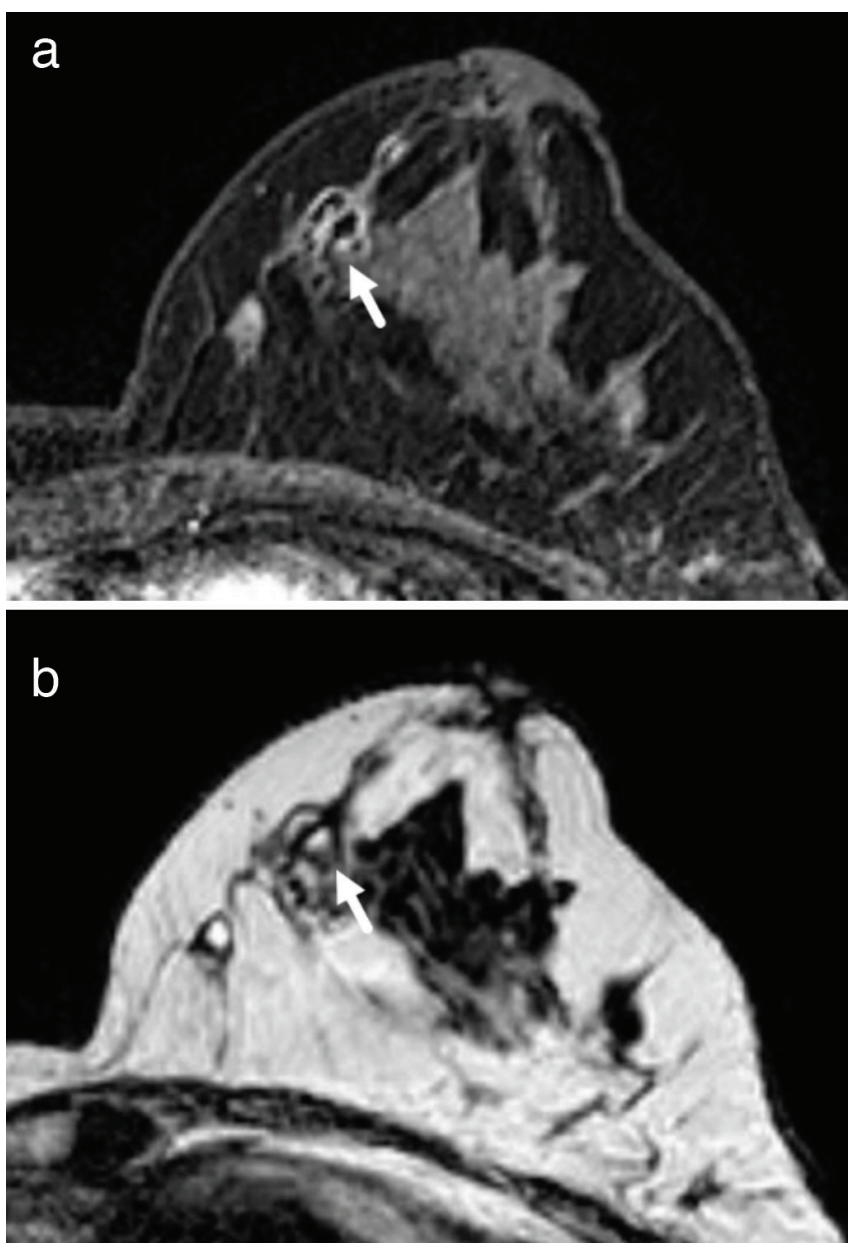

Figure 8. Fat necrosis seen with magnetic resonance imaging. (a) Rim enhancement (arrow) in the medial left breast on post-contrast T1-weighted image corresponds with (b) a fat-containing lesion (arrow) seen on T1-weighted pre-contrast image, both confirming fat necrosis 

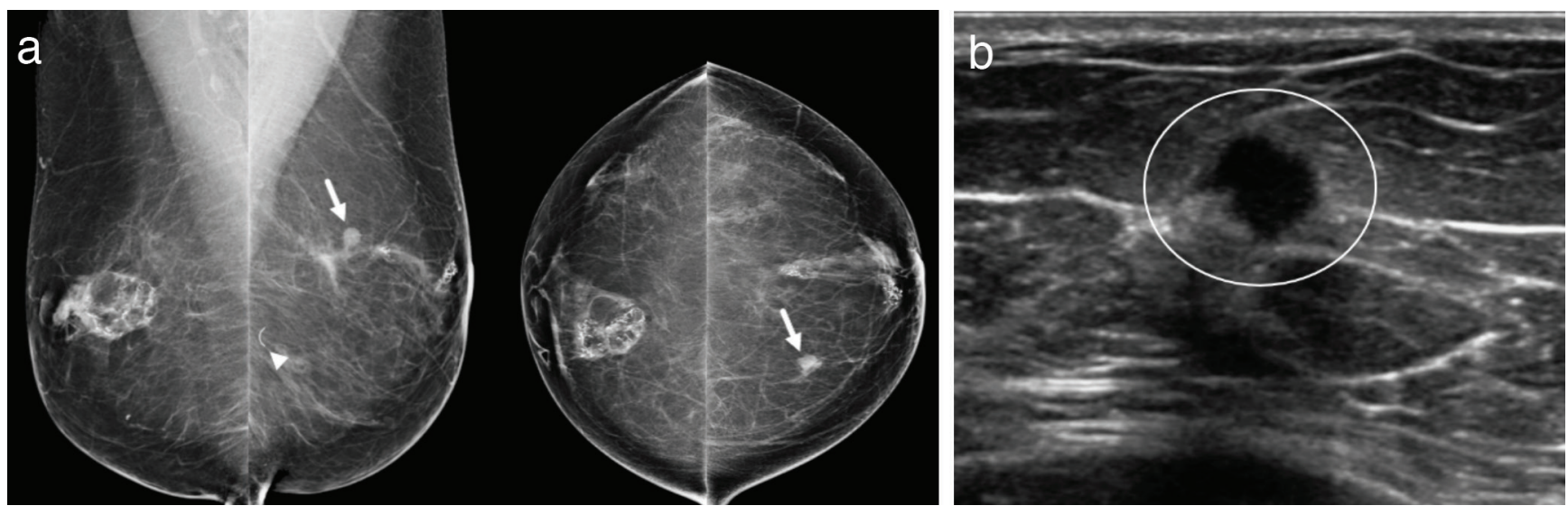

Figure 9. Interval cancer development following reduction mammoplasty. (a) This 58-year-old woman was found to have a new mass (arrows) in the 11 o'clock position of the left breast, middle depth, on routine screening mammography. Reduction changes, including areas of fat necrosis, are seen bilaterally. A suture needle (arrowhead) from the prior reduction surgery is noted posteriorly on the left mediolateral oblique projection. (b) Ultrasound imaging confirmed an irregularly shaped hypoechoic mass with indistinct margins (circle), with subsequent ultrasound-guided biopsy yielding triple-negative invasive ductal carcinoma

there is excessive degree of tissue fibrosis and no discernible internal fat. The enhancement kinetics for fat necrosis are highly variable, with a wide range of kinetic curves reported in the literature (13). Depending on the degree of diagnostic certainty, some lesions may be allowed short-term follow-up. However, if there is moderate to high clinical suspicion of malignancy, percutaneous sampling should be recommended.

Calcifications in the postoperative breast may also pose a diagnostic dilemma. Over time, benign calcifications caused by surgical changes tend to coarsen and become more dystrophic. As calcifications begin to form, they may appear amorphous and coarse heterogeneous within the BI-RADS $4 \mathrm{~b}$ intermediate suspicion category (17). Moreover, calcifications with a high likelihood of benignity $(\leq 2 \%$ risk of malignancy) may be classified as probably benign and followed as a precautionary measure. Any calcifications that remain suspicious after thorough mammographic work-up and/or follow-up should be subjected to stereotactic biopsy for histopathologic diagnosis (Figure 10).

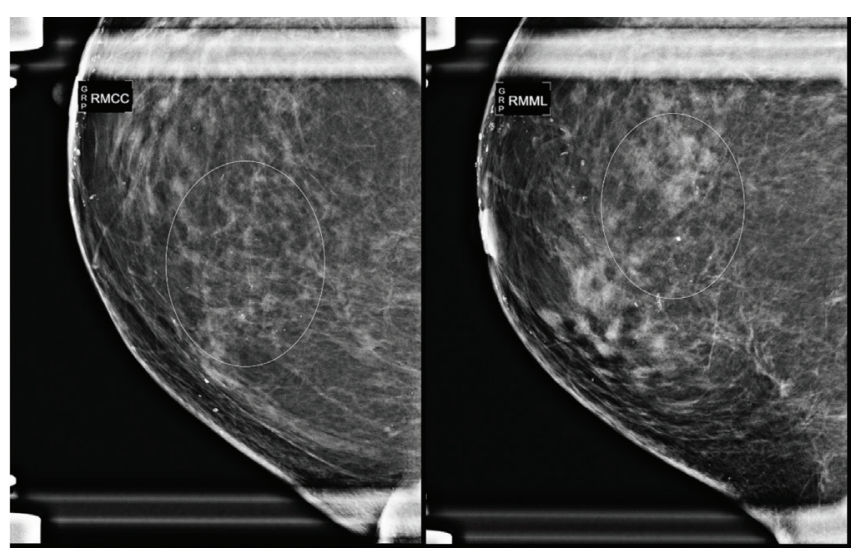

Figure 10. Developing malignant calcifications after reduction mammoplasty. This 66-year-old patient developed grouped, amorphous calcifications (circles) in the 3 o'clock position of the right breast 11 years after reduction mammoplasty, demonstrated on spot magnification diagnostic mammogram

\section{Conclusion}

All common breast imaging modalities reliably predict the imaging features of reduction mammoplasty. To avoid confusion with developing malignancy, radiologists interpreting these studies should be able to recognize the described patterns of distortion, scarring, and calcification. When there are indeterminate lesions, a biopsy or close short-term follow-up should be performed.

Peer-review: Externally and internally peer-reviewed.

\section{Author Contributions}

Surgical and Medical Practices: S.H.G., M.A.W., S.J.S.; Conception: S.H.G., M.A.W., S.J.S.; Design: S.H.G., M.A.W., S.J.S.; Analysis and/or Interpretation: S.H.G., M.A.W., S.J.S.; Literature Search: S.H.G., M.A.W., S.J.S.; Writing: S.H.G., M.A.W., S.J.S.

Conflict of Interest: The authors have no conflicts of interest to declare.

Financial Disclosure: The authors declared that this study has received no financial support.

\section{References}

1. American Society of Plastic Surgeons. 2018 Plastic Surgery Statistics Report 2019. Last Accessed Date: 21.04.2020. Available from: https:// www.plasticsurgery.org/documents/News/Statistics/2018/plastic-surgerystatistics-full-report-2018.pdf [Crossref]

2. Davis GM, Ringler SL, Short K, Sherrick D, Bengtson BP. Reduction mammaplasty: long-term efficacy, morbidity, and patient satisfaction. Plast Reconstr Surg 1995; 96: 1106-1110. (PMID: 7568486). [Crossref]

3. Glatt BS, Sarwer DB, O'Hara DE, Hamori C, Bucky LP, LaRossa D. A retrospective study of changes in physical symptoms and body image after reduction mammaplasty. Plast Reconstr Surg 1999; 103: 76-82; discussion 3-5. (PMID: 9915166). [Crossref]

4. Azurin DJ, Fisher J, Maxwell GP. Mastopexy. In: Weinzweig J, editor. Plastic Surgery Secrets Plus. Chicago, IL: Mosby/Elsevier; 2010. p. 453457.

5. Miller CL, Feig SA, Fox JWt. Mammographic changes after reduction mammoplasty. AJR Am J Roentgenol 1987; 149: 35-38. (PMID: 3495989). [Crossref] 
6. Brown FE, Sargent SK, Cohen SR, Morain WD. Mammographic changes following reduction mammaplasty. Plast Reconstr Surg 1987; 80: 691-698. (PMID: 3671561). [Crossref]

7. Danikas D, Theodorou SJ, Kokkalis G, Vasiou K, Kyriakopoulou K. Mammographic findings following reduction mammoplasty. Aesthetic Plast Surg 2001; 25: 283-285. (PMID: 11568832). [Crossref]

8. Margolis NE, Morley C, Lotfi P, Shaylor SD, Palestrant S, Moy L, et al. Update on imaging of the postsurgical breast. Radiographics 2014; 34: 642-660. (PMID: 24819786). [Crossref]

9. Burk KS, Seiler SJ, Porembka JH. Diagnosis, management, and percutaneous sampling of nipple-areolar calcifications: how radiologists can help patients avoid the operating room. AJR Am J Roentgenol 2021; 216: 48-56. (PMID: 33170739). [Crossref]

10. Hogge JP, Robinson RE, Magnant CM, Zuurbier RA. The mammographic spectrum of fat necrosis of the breast. RadioGraphics 1995; 15: 13471356. (PMID: 8577961). [Crossref]

11. Shaheen R, Schimmelpenninck CA, Stoddart L, Raymond H, Slanetz PJ. Spectrum of diseases presenting as architectural distortion on mammography: multimodality radiologic imaging with pathologic correlation. Semin Ultrasound CT MR 2011; 32: 351-362. (PMID: 21782125). [Crossref]
12. Cakir M, Kucukkartallar T, Tekin A, Selimoglu N, Poyraz N, Belviranli $\mathrm{MM}$, et al. Comparison of mammography sensitivity after reduction mammoplasty targeting the glandular and fat tissue. Ulus Cerrahi Derg 2015; 31: 68-71. (PMID: 26170752). [Crossref]

13. Daly CP, Jaeger B, Sill DS. Variable appearances of fat necrosis on breast MRI. AJR Am J Roentgenol 2008; 191: 1374-1380. (PMID: 18941072). [Crossref]

14. Kerrigan CL, Slezak SS. Evidence-based medicine: reduction mammaplasty. Plast Reconstr Surg 2013; 132: 1670-1683. (PMID: 24281593). [Crossref]

15. Tang R, Acevedo F, Lanahan C, Coopey SB, Yala A, Barzilay R, et al. Incidental breast carcinoma: incidence, management, and outcomes in 4804 bilateral reduction mammoplasties. Breast Cancer Res Treat 2019; 177: 741-748. (PMID: 31317348). [Crossref]

16. Muir TM, Tresham J, Fritschi L, Wylie E. Screening for breast cancer post reduction mammoplasty. Clin Radiol 2010; 65: 198-205. (PMID: 20152275). [Crossref]

17. Tan PH, Lai LM, Carrington EV, Opaluwa AS, Ravikumar KH, Chetty N, et al. Fat necrosis of the breast--a review. Breast 2006; 15: 313-318. (PMID: 16198567). [Crossref] 